Cela, F. (2018). Green's Relations in Rings and Completely Simple Rings. Journal of Advances in Mathematics, 14(2), 7965-7974. https://doi.org/10.24297/jam.v14i2.7781. This work is licensed under a Creative Commons Attribution 4.0 International License.

\title{
GREEN'S RELATIONS IN RINGS AND COMPLETELY SIMPLE RINGS
}

\section{Florion ÇELA and Petraq PETRO}

Faculty of Natural and Sciences. University of Tirana. florion.cela@fshn.edu.al, petraq.petro@yahoo.com

\begin{abstract}
In this paper we prove that which of Green's relations $\mathcal{L}, \mathcal{R}, \mathcal{H}$ and $\mathcal{D}$ in rings preserve the minimality of quasi-ideal. By this it is possible to show the structure of the classes generated by the above relations which have a minimal quasi ideal. For the completely simple rings we show that they are generated by the union of zero with a $\mathcal{D}$-class. Also we emphasize that a completely simple ring coincides with the union of zero with a $\mathcal{D}$-class if and only if it is a division ring.

Keywords: Rings, Semigroups, Green's relation, quasi-ideal, completely simple ring.

Subject Classification: 16-XX, 20M25

Website: https://cirworld.com
\end{abstract}

\section{Introduction}

By a ring we mean a ring, which does not neccessarily have a identity. Green's relations are successful tools for studying semigroups. In an analogous way the relations $\mathcal{L}, \mathcal{R}, \mathcal{H}$ and $\mathcal{D}$ are introduced and studied also in rings and are called Green's relations in rings [6]. Green's relations in rings serve not only to study rings but also they are tools to point out the general connection between rings and semigroups, and especially the connection between rings and their multiplicative semigroups.

In this paper by using Green's Lemma for relation $\mathcal{L},[\mathcal{R}]$ we prove that for each to elements $a, b$ of a ring $A$ which are $a \mathcal{L} b[a \mathcal{R} b]$ the quasi ideal $(a)_{q}$ is minimal if and only if $(b)_{q}$ is a minimal quasi ideal. This implies that if two elements $a, b$ of a ring $A$ are $a \mathcal{H} b[a \mathcal{D} b]$, the quasi ideal $(a)_{q}$ is minimal if and only if $(b)_{q}$ is a minimal quasi ideal. After this we show the structure of $H$-class which contain a minimal quasi ideal,by pointing out that such a $H$-class is a union of minimal quasi ideals, which as rings are isomorphic with each other. Also we can only show that a $\mathcal{L}[\mathcal{R}, \mathcal{D}]$ class that contains a minimal quasi-ideal is a union of minimal quasi-ideals and each two of them are isomorphic as additive groups.

For the completely simple rings firstly we show that $A$ be a completely simple ring, then there exists an idempotent element $e \in A$ such that $A e$ is a left minimal ideal, $e A$ is a right minimal ideal and $A=A$ e.e $A$. Then we prove that in a 
completely simple ring $A$ there exists a $\mathcal{D}$ - class $D$ of $A$ such that the subring obtained by $D \cup\{0\}$ coincides with $A$. Lastly we show that in a completely simple ring $A$ the equality $A=D_{e} \cup\{0\}$, where $e=e^{2}$, is satisfied if and only if $A$ is a division ring.

\section{Preliminares}

We give some notions and present some auxiliary results that will be used throughout the paper. All unexplained concepts and proposition the reader may find to [1],[4],[7].

Let $(A,+,$.$) be a ring and B, C$ two subsets of $A$.

We write:

$$
\begin{gathered}
B+C=\{b+c \in A \mid b \in B, c \in C\}, \\
B C=\left\{\sum_{i=1}^{n} b_{i} c_{i} \in A \mid \text { for every natural number } n \in \mathbb{N} ; b_{i} \in B, c_{i} \in C\right\},
\end{gathered}
$$

For the sake of simplicity we write $b+C, b C, B c$ instead of $\{b\}+C,\{b\} C, B\{c\}$. The relations $\mathcal{R}, \mathcal{L}$ and $\mathcal{H}$ in a ring $A$ are defined as following [6]:

$$
\begin{gathered}
\forall(a, b) \in A^{2}, a \mathcal{R} b \Leftrightarrow(a)_{r}=(b)_{r}, \\
\forall(a, b) \in A^{2}, a \mathcal{L} b \Leftrightarrow(a)_{l}=(b)_{l}, \\
\forall(a, b) \in A^{2}, a \mathcal{H} b \Leftrightarrow(a)_{r}=(b)_{r} \text { and }(a)_{l}=(b)_{l},
\end{gathered}
$$

where $(a)_{r},(b)_{r},(a)_{l},(b)_{l}$ are respectively right principal ideals, left principal ideals generated by the elements $a, b$ of $A$. The relations $\mathcal{R}, \mathcal{L}$ and $\mathcal{H}=\mathcal{R} \cap \mathcal{L}$ are equivalence relations. The equivalence classes of an element $a \in A$ are denoted respectively $R_{a}, L_{a}$ and $H_{a}$.

In [6] it is shown that for each ring $A, \mathcal{R} \circ \mathcal{L}=\mathcal{L} \circ \mathcal{R}$. So we get the Green's relation $D=\mathcal{R} \circ \mathcal{L}=\mathcal{L} \circ \mathcal{R}$ in rings. The equivalence class of an element $a \in A$ respect to the relation $\mathcal{D}$ is denoted $D_{a}$.

Let $(A,+,$.$) be a ring . A subgroup Q$ of additive group $(A,+)$ of this ring is called quasi-ideal if $A Q \cap Q A \subseteq Q$ [7].

The intersection of all the quasi ideals that contain the element $a \in A$ is called principal quasi-ideal generated by the element $a$ and is denoted $(a)_{q}$.

It is not difficult to prove that for each element $a$ of a ring $A$ the following equalities hold :

$$
\begin{gathered}
(a)_{r}=\mathbb{Z} a+a A, \\
(a)_{l}=\mathbb{Z} a+A a, \\
(a)_{q}=\mathbb{A} a+A a \cap a A .
\end{gathered}
$$

For each integer $z \in \mathbb{Z}$ and for each three elmenents $a, x, y$ of a ring $A$ we denote :

$$
\begin{aligned}
& a(z, x)=z a+a x \\
& (z, y) a=z a+y a .
\end{aligned}
$$


Leme 2.1 (Green's Lemma for the relation $\mathcal{L}$ )[6]. Let $a, b$ be two elements of a ring A such that $a \mathcal{L} b$.

If $b=(z, y) a$ and $a=\left(z^{\prime}, y^{\prime}\right) b$, then the maps :

$$
\begin{gathered}
f:(a)_{r} \rightarrow(b)_{r}, f(c)=(z, y) c, \\
g:(b)_{r} \rightarrow(a)_{r}, f(d)=\left(z^{\prime}, y ;\right) d,
\end{gathered}
$$

are inverse with each other, preserve the $\mathcal{L}$-classes and maps bijectively $R_{a}$ to $R_{b}$ and $R_{b}$ to $R_{a}$ respectively.

The dual of Lemma 2.1 for the Green's relation $\mathcal{R}$ is formulated in an analogous manner. A non-zero two-side right ideal (left ideal,quasi-ideal) $B$ of a ring $A$ (subgroup with zero) is called minimal (0-minimal) if $B$ does not properly contain any non-zero right ideal (left ideal,quasi-ideal).

By using the Green's relation $\mathcal{H}$, the Proposition 1 [7] can be formulated as follows:

Proposition 2.1 A quasi-ideal $Q$ if the ring $A$ is minimal if and only if the $Q-\{0\}$ is included in an $\mathcal{H}$-class

Theorem 2.1 [Green's Theorem for rings][6]. If the elements $a, b, a b$ of a ring $(A,+,$.$) all belong to the some \mathcal{H}$-class $H$ of $A$, then $H$ is a subgroup of the multiplicative semigroup $(A,$.$) of the ring (A,+,$.$) .$

Definition 2.1 [3] A ring $A$ is called completely simple ring if it is simple and have at least one minimal left or right ideal.

Definition 2.2 [3] $A$ ideal $M$ of a ring $A$ is called completely simple ideal of $A$ if $M$ is a completely subring of $A$.

Proposition 2.2 [3] If $M$ is a completely simple ideal of a a ring $A$, then $M=L R$, where $L$ and $R$ are minimal left and right ideals of $A$, respectively and $R L \neq 0$.

Definition 2.3 [1] A semigroup with zero $S$ is called completely 0-simple if it is a 0-simple semigroup and it have at least one 0-minimal left ideal and at least one 0-minimal right ideal.

Definition 2.4 [5] Let $(A,+,$.$) be a ring. If the multiplicative semigroup (A,$.$) of$ this ring is completely 0 -simple, then $(A,+,$.$) is a division ring.$

\section{Main Results}

Let $A$ be a ring and $a$ an element of $A$ such that $(a)_{q}$ is a minimal quasi ideal. Does the Green's relation $\mathcal{L}$ preserve the minimality of the quasi-ideal $(a)_{q}$ ? The answer to this question is positive. The following theorem holds :

Theorem 3.1 Let $a, b$ be two elements of the ring $A$ such that $a \mathcal{L} b$. The quasi ideal $(a)_{q}$ is minimal if and only if $(b)_{q}$ is a minimal quasi ideal. 
Proof. Since $a \mathcal{L} b$, then there exist integers $z, z^{\prime}$ and elements $y, y^{\prime}$ of $A$ such that the following equalities hold:

$$
b=(z, y) a, a=\left(z^{\prime}, y\right) b .
$$

We denote $\lambda:(a)_{q} \rightarrow(b)_{q}$ such that

$$
\forall c \in(a)_{q}, \lambda(c)=(z, y) c .
$$

Firstly we show that the map $\lambda$ is well defined. So we need to show that for each element $c$ of $(a)_{q}$ the element $(z, y) c$ is an element of the quasi ideal $(b)_{q}$. For the element $c \in(a)_{q}$ there exist an integer $z$, and elements $x_{1}, x_{1}^{\prime}$ of $A$ such that $c=z_{1} a+a x_{1}=z_{1} a+x_{1}^{\prime} a$. The following equalities hold:

$$
\begin{gathered}
(z, y) c=(z, y)\left(z_{1} a+a x_{1}\right)=z\left(z_{1} a+a x_{1}\right)+y\left(z_{1} a+a x_{1}\right)= \\
z_{1}(z a+y a)+(z a+y a) x_{1}=z_{1}((z, y) a)+((z, y) a) x_{1}=z_{1} b+b x_{1} \\
(z, y) c=(z, y)\left(z_{1}+x_{1}^{\prime} a\right)=z\left(z_{1} a+x_{1}^{\prime} a\right)+y\left(z_{1} a+x_{1}^{\prime} a\right)=z_{1}(z a+y a)+\left(z x_{1}^{\prime}+y x_{1}^{\prime}\right) a= \\
z_{1}(z, y) a+\left((z, y) x_{1}^{\prime}\right) a=z_{1} b+\left((z, y) x_{1}^{\prime}\right)\left(z_{1}^{\prime}, y^{\prime}\right) b=z_{1} b\left((z, y) x_{1}^{\prime}\left(z^{\prime}, y^{\prime}\right)\right) b,
\end{gathered}
$$

which show that

$$
(z, y) c \in \mathbb{Z} b+b A \cap A b=(b)_{q} .
$$

Since $b \mathcal{L} a$, in a similar way we can prove that the map $\lambda^{\prime}:(b)_{q} \rightarrow(a)_{q}$

$$
\forall d \in(b)_{q}, \lambda^{\prime}(d)=\left(z^{\prime}, y^{\prime}\right) d,
$$

is well defined.

By using Green's Lemma for the relation $\mathcal{L}$ [Lemma 2.1] the maps :

$$
\begin{gathered}
f:(a)_{r} \rightarrow(b)_{r}, \forall c \in(a)_{r}, f(c)=(z, y) c, \\
g:(b)_{r} \rightarrow(a)_{r}, \forall d \in(b)_{r}, g(d)=\left(z^{\prime}, y^{\prime}\right) d,
\end{gathered}
$$

are inverse with each other, preserve $\mathcal{L}$-classes and map bijectively $R_{a}$ in $R_{b}$ and $R_{b}$ in $R_{a}$ respectively. Thus, we get that also the maps $\lambda$ and $\lambda^{\prime}$ are inversive with each other and since :

$$
\begin{aligned}
& \forall c \in(a)_{q}, \lambda(q)=f(q), \\
& \forall c \in(b)_{q}, \lambda^{\prime}(q)=g(c) .
\end{aligned}
$$

we have

$$
\forall c \in(a)_{q}, c \mathcal{L} \lambda(c) .
$$

Now we suppose that $(a)_{q}$ is a minimal quasi-ideal. By using the Propositon 2.1 we get that $(a)_{q}-\{0\} \subseteq H_{a}$. So, since

$$
\forall c \in(a)_{q}-\{0\}, b=\lambda(a) \mathcal{R} \lambda(c),
$$

the following proposition holds

$$
\forall c \in(a)_{q}-\{0\}, b \mathcal{H} \lambda(c) .
$$


Thus

$$
\lambda\left((a)_{q}-\{0\}\right) \subseteq H_{b},
$$

and consequently, since $\lambda$ is a bijective map we have that :

$$
(b)_{q}-\{0\}=\lambda\left((a)_{q}-\{0\}\right) \subseteq H_{b} .
$$

So, from the Proposition 2.1 the quasi-ideal $(b)_{q}$ is minimal.

If the quasi-ideal $(b)_{q}$ is minimal, by using the fact that $b \mathcal{L} a$, and what we proved above we get that also $(a)_{q}$ is a minimal quasi ideal.

Analogously by using Green's Lemma for the relation $\mathcal{R}$, the following theorem can be proved:

Theorem 3.2 Let $a, b$ be two elements of a ring $A$ such that $a \mathcal{R} b$. The quasi-ideal $(a)_{q}$ is minimal if and only if the quasi ideal $(b)_{q}$ is minimal.

Immediately from therorems 3.1, 3.2 we have the following corollary :

Corollary 3.1 Let $a, b$ be two elements of a ring $A$ such that $a \mathcal{H} b$. The quasi-ideal $(a)_{q}$ is minimal if and only if the quasi ideal $(b)_{q}$ is minimal.

Since, where for each two element $a, b$ of a ring $A$ we have $a \mathcal{D} b$ there exists an element $c \in A$ such that $a \mathcal{L} c$ and $c \mathcal{R} b$, by using at first Theorem 3.1 and then Theorem 3.2 we have :

Theorem 3.3 Let $a, b$ be two elemnets of a ring $A$ such that $a \mathcal{D} b$. The quasi-ideal $(a)_{q}$ is minimal if and only if the quasi ideal $(b)_{q}$ is minimal.

The following theorema shows the structure of $\mathcal{H}$-class that contains a minimal quasi ideal.

Theorem 3.4 Let $Q$ be a minimal quasi ideal of a ring $A$, then the $\mathcal{H}$-class $H$ which contain $Q-\{0\}$ such that $H \cup\{0\}$ is a union of minimal quasi ideals, that as rings are isomorphic with each other.

Proof. Let $a$ be an elment of $Q$ different from zero. It is evident that $(a)_{q}=$ $Q$. We denote $H$, the $\mathcal{H}$-class that contains the quasi-ideal $Q=(a)_{q}$, which we know that it exist from Proposition 2.1. Since $a \in H$, then the following equality $H=H_{a}$ hold. For each element $b \neq 0$ of $H=H_{a}$ we have $b \mathcal{H} a$ and by using Corollary 3.1 we get the minimality of the quazi-ideali $(b)_{q}$. So,

$$
(b)_{q}-\{0\} \subseteq H_{b}=H_{a}=H .
$$

Thus, $(b)_{q} \subseteq H \cup\{0\}$, and consequently we get the equality $H \cup\{0\}=\cup_{b \in H}(b)_{q}$, wich show that the $\mathcal{H}$-class $H$ it is such that $H \cup\{0\}$ is the union of minimal quasi-ideals that are contained in it. To prove the rest of the theorem we see the two possible cases :

First case. It exists an element $c \in H$ such that the quasi ideal $(c)_{q}$ is with nonzero multiplication. Then it is known that $(c)_{q}$ is a division ring. We denote by $e$ the identity element of this division ring, then $e \in H$ because $(c)_{q}-\{0\} \subseteq H$. 
Hence by Green's Theorem for rings [Theorema 2.1] $H$ is a group.Then, since $(c)_{q} \subseteq H \cup\{0\}$ we get $(c)_{q}=H \cup\{0\}$. Moreover for each element $b \in H$ it is true that $(b)_{q}=(c)_{q}$, which show that we have only one minmal quasi-ideal that is contained in $H \cup\{0\}$. So we can say that $H \cup\{0\}$ is a union of minimal quasiideals which they are also isomorphic with each other.

Second case. For each element $c \in H$ the quasi-ideal $(c)_{q}$ is with zero multiplication . Let $c_{1}$ be an element of $H$. For this element we have that $c_{1} \mathcal{L} c$. Then there exist the integer $z$ and the element $y \in A$ such that $c=(z, y) c_{1}$. In the same way as in the proof of Theorem 3.1 we get that $\lambda_{1}:\left(c_{1}\right)_{q} \rightarrow(c)_{q}$ such that

$$
\forall x \in\left(c_{1}\right)_{q}, \lambda_{1}(x)=(z, y) x=z x+y x
$$

is a map which has an inversive map, and consequently it is a bijective map . For each two element $x_{1}, x_{2}$ of $\left(c_{1}\right)_{q}$ the following equalities hold :

$$
\begin{gathered}
\lambda_{1}\left(x_{1}+x_{2}\right)=(z, y)\left(x_{1}+x_{2}\right)=z\left(x_{1}+x_{2}\right)+y\left(x_{1}+x_{2}\right)= \\
=\left(z x_{1}+y x_{1}\right)+\left(z x_{2}+y x_{2}\right)=\lambda_{1}\left(x_{1}\right)+\lambda_{1}\left(x_{2}\right), \\
\lambda_{1}\left(x_{1} x_{2}\right)=\lambda_{1}(0)=(z, y) 0=0=\lambda_{1}\left(x_{1}\right) \cdot \lambda_{1}\left(x_{2}\right),
\end{gathered}
$$

which show that the bijection $\lambda_{1}$ is in fact an isomorphism of the minimal quasi-ideal $\left(c_{1}\right)_{q}$ to the arbitrary minimal quasi-ideal $(c)_{q}$ which is contained in $H \cup\{0\}$. Hence each two minimal quasi-ideals which are contained in $H \cup\{0\}$ are isomorphic rings with each other .

It is natural to raise the following question : Does analogous theorems as Theorem 3.4 hold also for Green's relations $\mathcal{L}, \mathcal{R}, \mathcal{D}$ in rings? However for Green's relations $\mathcal{L}, \mathcal{R}, \mathcal{D}$ in rings there are true some "weaker" theorems. More precisely we have the following theorem :

Theorem 3.5 Let $Q$ be a minimal quasi-ideal of the ring $(A,+,$.$) , then there exist$ $\mathcal{L},[\mathcal{R}, \mathcal{D}]$ class $L[R, D]$ which contains the minimal quasi-ideal $Q$. Moreover the $\mathcal{L},[\mathcal{R}, \mathcal{D}]$-class $L[R, D]$ is such that $L \cup\{0\}(R \cup\{0\}, D \cup\{0\})$ is a union of quasi-ideals, which as additive groups are isomorphic with each other.

Proof. We only show the proof for the $\mathcal{L}$-class $L$, because the proof is similar for the $\mathcal{R}$-class $R$ and the $\mathcal{D}$-class $D$.

Let $Q$ be a minimal quasi-ideal and let $a$ be a nonzero element of $Q$. Then $Q=(a)_{q}$ and the Proposition 2.1 implies that $Q-\{0\} \subseteq H_{a}$. Since $H_{a} \subseteq L_{a}, \mathcal{L}$ class $L=L_{a}$ contains $Q-\{0\}$. For each element $b \neq 0$ of $L=L_{a}$, we have $b \mathcal{L} a$ and from Theorem 3.1 we get that the quasi-ideal $(b)_{q}$ is minimal.

From the Proposition 2.1 we have that:

$$
(b)_{q}-\{0\} \subseteq H_{b} \subseteq L_{b} \subseteq L_{a}=L .
$$

So, $(b)_{q} \subseteq L$ and consequently the following equality holds

$$
L \cup\{0\}=\cup_{b \in L}(b)_{q},
$$


which shows that $\mathcal{L}$-class $L$ is such that $L \cup\{0\}$ is a union of minmal quasiideals.

Let $c_{1}$ be an element of $L$. For this arbitrary element $c \in L$ we have that $c_{1} \mathcal{L} c$. The rest of the proof is the same as the proof of the second case of Theorem 3.4, of course by considering the quasi ideals $\left(c_{1}\right)_{q}$ and $(c)_{q}$ as additive groups of the ring $(A,+,$.$) .$

If we repeat the proof of Proposition 2.2 for the case $M=A$ we get this:

Proposition 3.1 Let $A$ be a completely simple ring, then it exists the element $e \in A$ such that Ae is a left minimal ideale, $e A$ is a right minimal ideal and $A=A e . e A$

Now to find a connection between completely simple rings and Green's relation $\mathcal{D}$ in ring, we use the following lemma.

Leme 3.1 A right [left] ideal $R[L]$ of a ring $A$ is minimal if and only if for each $a \neq 0$ of $R[L]$ we have:

$$
R=R_{a} \cup\{0\}\left(L=L_{a} \cup\{0\}\right) .
$$

We omit the proof, because it is straightforward.

Theorem 3.6 Let $A$ be a completely simple ring then there exists a $\mathcal{D}$-class $D$ of $A$ such that the ring $A$ is generated by $D \cup\{0\}$.

Proof. By Proposition 3.1 we have $A=A e . e A$, where $e^{2}=e, A e$ is a left minimal ideal and $e A$ is a right minimal ideal. By applying Lemma 3.1 we get :

$$
A e=L_{e} \cup\{0\}, e A=R_{e} \cup\{0\} .
$$

Hence the following equalities hold:

$$
A=A e . e A=\left(L_{e} \cup\{0\}\right)\left(R_{e} \cup\{0\}\right)=L_{e} R_{e} \cup\{0\} .
$$

Since $L_{e} \subseteq D_{e}$ and $R_{e} \subseteq D_{e}$ we have:

$$
A=L_{e} R_{e} \cup\{0\} \subseteq<D_{e} \cup\{0\}>\subseteq A,
$$

where $<D_{e} \cup\{0\}>$ is a subring of $A$ generated by the union of $\mathcal{D}$-class $D_{e}$ with $\{0\}$. So, $A=<D_{e} \cup\{0\}>$.

By the above theorem is it is natural to ask the following question : Let $A$ be a completely simple ring and $e$ an idempotent element in $A$, then in what conditions the equality $A=D_{e} \cup\{0\}$ holds? The answer to this question is given by the following proposition:

Proposition 3.2 Let $(A,+,$.$) be a completely simple ring and e an idempotent ele-$ ment of $A$. Then $A=D_{e} \cup\{0\}$ if and only if $(A,+,$.$) is a division ring.$

Proof. It is obvious where that $(A,+,$.$) is a division ring, then, if we denote by$ $e$ be the identity element of $A$ then $A=D_{e} \cup\{0\}$.

Conversely, let suppose that there exist an idempotent element $e$ of the completely simple ring $(A,+,$.$) such that A=D_{e} \cup\{0\}$. It is not difficult to show 
that the multiplicative semigroup $(A,$.$) of the ring (A,+,$.$) is completely sim-$ ple. Now by applying Propositon 2.4 the completely simple ring $(A,+,$.$) is a$ division ring.

\section{References}

[1] Clifford, A.H. and Preston, G.B. The algebraic theory of semigroups. Vol.I. Amer.Math.Soc.,Providence,R.I, 1961.

[2] Green, J.M. On the structure of semigroups. Annals of Mathematics. 54(1)(1961),163-172.

[3] Gluskin,L.M. and Steinfeld, O. Rings (semigroups) containing minimal (0minimal) right and left ideals. Publ.Math.(Debrecent), 25(1978), 275-280.

[4] Howie,J.M. Fundamentals of semigroups theory. Oxford Universit Press (1995).

[5] Lallement, G. Demi-groups reguliares, Ann.Mat.Pura Appl.(4)77(1969),47129.

[6] Petro,P. Green's relations and minimal quasi-ideals, communication in Algebra. 30(10)(2002), 4677-4686.

[7] Steinfeld, O. Quasi-ideals in rings and semigroups. Akademiai Kiado, Budapest, 1978.

[8] Stewart, P.N Quasi ideals in rings. Acta Math.Acad.Sci.Hunga. 38 (1981), 231-235. 